Original

\title{
Trastorno de adaptación. Análisis de la Incapacidad Laboral por contingencias comunes en Ibermutuamur, Mutua de Accidentes de Trabajo y Enfermedades Profesionales de la Seguridad Social
}

\author{
Adjustment disorders. Analysis of sickness absence episodes caused by non- \\ work-related diseases or accidents at Ibermutuamur, Mutual Insurance Compa- \\ ny for Occupational Accidents and Diseases, Social Security
}

\section{Rosa Carmen Enguita Martín ', Rafael Ramos Muñoz ${ }^{2}$}

\section{Médico. Jefe de Departamento de Administración Sanitaria. Dirección Médica de contingencias comunes de Ibermutuamur. Madrid. España. \\ 2. Director Médico de contingencias comunes de Ibermutuamur. Madrid. España.}

Recibido: 29-05-13

Aceptado: 15-07-13

\author{
Correspondencia \\ Rosa Carmen Enguita Martín \\ Paseo de la Castellana, 157 10.' \\ 28046 - Madrid. (España). \\ Teléfono: 658802796 \\ Correo electrónico: rcenguita@hotmail.com
}

Resumen

Las enfermedades mentales constituyen una importante causa de Incapacidad Temporal y absentismo laboral en los países industrializados, con la consiguiente carga socio-sanitaria e impacto económico que conllevan. Una de las causas más importante de estas enfermedades son los Trastornos de Adaptación. La predisposición o vulnerabilidad individual que da lugar a su aparición y manifestaciones, junto con la dificultad de aplicar criterios diagnósticos determinantes y cuantificables, hacen que muchos pacientes sean encuadrados en este diagnóstico y no reciben un tratamiento adecuado a la patología que realmente padecen, con el consiguiente desajuste temporal en la duración de su situación de Incapacidad Laboral.

Por este motivo se inicia la revisión del comportamiento de la Incapacidad Temporal de los trabajadores controlados por contingencias comunes en Ibermutuamur con el diagnóstico de Trastorno de Adaptación durante los años 2008 al 2010, valorar los resultados y concluir si alguna variable permitirá mejorar el control y seguimiento de estos procesos, ajustando su duración en beneficio del paciente, su empresa, la Mutua y la sociedad en general.

Tras la realización del estudio epidemiológico observacional de los datos registrados en los procesos de nuestro propio programa sanitario durante los años mencionados, podemos concluir que, salvo algunos datos orientativos para su gestión, como su mayor frecuencia de aparición en mujeres, y en ellas, de recaídas y evolución a Incapacidad Permanente, o el hecho de que además del diagnóstico genérico, los subtipos diagnósticos "Reacción de adaptación con características emocionales mixtas" y "Reacción de adaptación con humor de ansiedad" son los más frecuentes y los que se repiten en frecuencia en casi todos los resultados de la valoración del resto de las variables del estudio, no existe ninguna variable que por sí misma pueda determinar la toma de decisiones para mejorar la gestión y evolución de la Incapacidad Temporal por esta patología. 
Es primordial reseñar que uno de los inconvenientes más importantes encontrados ha sido la falta de codificación de este diagnóstico en sus diferentes subtipos diagnósticos, lo que puede determinar un déficit de información clave para la correcta adscripción diagnóstica de los pacientes y posterior tratamiento, repercutiendo en la evolución y duración de la Incapacidad Temporal y el absentismo laboral.

Med Segur Trab (Internet) 2013; 59 (232) 322-344

Palabras clave: enfermedad mental, patología psiquiátrica, reacción de adaptación, incapacidad temporal.

Abstract

Mental illnesses are a major cause of sick leave and absenteeism in industrialized countries, resulting in a burden of socio-sanitary and economic impact. One of the most important related-causes are Adjustment Disorders. Individual predisposition or vulnerability leading to their appearance and symptoms, along with the difficulty of applying diagnostic criteria and quantifiable determinants, means that many patients are misclassified in the same diagnosis. Therefore, they do not receive adequate treatment for the disease that really suffer from, generating temporary mismatch in the duration of sick leave.

Due to all this, we started a review of the sick leave behavior from this disease in workers monitored for non-work-related diseases at Ibermutuamur between 2008 and 2010 in order to evaluate the results and to conclude whether some of the variables will improve the management and control of these processes by adjusting the length on behalf of the patients, their company, the mutual insurance company and the society in general.

Following the completion of the observational epidemiological study according to the data recorded in the processes in our own health program system during the period of time mentioned, we can conclude that, except for some key data for management, as the fact of their higher frequency in women, and in them, the relapses and progression to total disability or the fact that besides the generic diagnostic, subtypes "Adjustment reaction with mixed emotional features" and "Adjustment disorder with mixed anxiety and depressed mood" are the most common and frequently observed in almost all the assessment results of the other study variables, there is no variable itself which could determine the decision-making to improve the management of sickness absence from this disease.

It is essential to note that one of the major drawback found was the lack of coding of this disease in their diagnostic subtypes, which can determine a key information deficit to proper assignment of the pathology and the posterior treatment, thus affecting the evolution and the duration of sick leave and absenteeism.

Med Segur Trab (Internet) 2013; 59 (232) 322-344

Keywords: mental illness, psychiatric disorders, adjustment reaction, sick leave. 


\section{INTRODUCCIÓN}

Las enfermedades mentales son una de las principales causas de Incapacidad Temporal en nuestro país.

La relación de la Incapacidad Temporal con el absentismo laboral es un fenómeno que se viene produciendo desde que surgen las relaciones laborales basadas en el trabajo asalariado por cuenta ajena. Sus implicaciones económicas y sociales han dado lugar, en los países de mayor desarrollo industrial y donde se producen con creciente virulencia, a plantear reiteradamente la necesidad de aplicar medidas efectivas para paliar sus consecuencias y reducir su incidencia ${ }^{1}$.

La causa más frecuente de absentismo laboral en España es la enfermedad común. Dentro de las patologías que concentran el mayor número de incapacidades temporales y de mayor duración están las enfermedades psiquiátricas junto a las dolencias osteomusculares y los procesos oncológicos.

En España se han desarrollado una serie de estudios para evaluar la prevalencia de trastornos mentales entre los que destaca la participación española en el Estudio Europeo de la Epidemiología de los Trastornos Mentales (ESEMeD), estudio de carácter diagnóstico

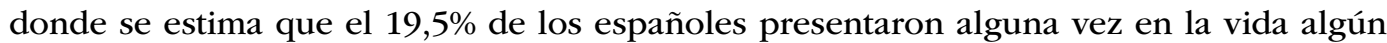
trastorno mental (prevalencia-vida), el 15,7\% de los hombres y el 22,9\% de las mujeres ${ }^{2}$.

También se registraron aproximadamente 5.6 millones de bajas laborales durante 2009 por enfermedad o accidentes, consumiendo casi 300 millones de jornadas de trabajo por este concepto y suponiendo un gasto de 8.143 millones de euros según datos del Ministerio de Trabajo e Inmigración (MTIN) ${ }^{3}$.

Según la Encuesta Trimestral de Costes laborales del Instituto Nacional de Estadística, las horas no trabajadas por incapacidad laboral en España por persona el año 2010 fueron 55. Se estima que el coste directo del absentismo por incapacidad temporal para el sector empleador en el año 2010 fue de 7.046 millones de euros, lo que representa el $0,74 \%$ del PIB $^{4}$.

Es difícil estimar el impacto económico provocado por la Incapacidad Temporal, puesto que, además de los días de trabajo perdidos, conlleva una serie de costes indirectos como los gastos de contratación de sustitutos, la disminución de la productividad para la empresa, aumento del gasto sanitario, disminución de la competitividad, disminución de la calidad de los servicios, etc.

La problemática en el control de las Incapacidades Temporales por contingencias comunes surge cuando algunos trabajadores tienden a prolongar su incapacidad temporal por motivos sociales, socio-familiares, laborales o por puro rentismo, teniendo en cuenta que las Mutuas no tienen la potestad de emitir el alta médica a los trabajadores.

La vida implica inevitables circunstancias que la mayoría de seres humanos aprenden a manejar adaptando sus respuestas bio-psicosociales para superarlas. Todo estímulo, situación o acontecimiento que impacte en un individuo y le obligue a esfuerzos de ajuste adaptativo constituye un acontecimiento estresante.

Los Trastornos de adaptación son básicamente reacciones muy intensas a un hecho estresante de la vida y se distinguen de la reacción normal ante el estrés porque sus componentes emocionales y conductuales resultan de mayor intensidad o son más incapacitantes de lo esperado.

Actualmente, para la clasificación de la patología psiquiátrica se emplean dos sistemas de clasificación diagnóstica: la CIE-10, Clasificación internacional de enfermedades, décima versión, correspondiente a la ICD, International Statistical Classification of Diseases and Related Health Problems (Organización Mundial de la Salud, 1992) y el DSM-IV Diagnostic and Statistical Manual of Mental Disorders cuarta 
edición (Asociación Psiquiátrica Americana (APA, 1994). DSM-IV TR (texto revisado) (APA, 2000) $)^{5,6}$.

No existe un sistema clasificatorio único, y aunque la CIE-10 ya está disponible, no ha entrado en vigor su uso a nivel internacional, por lo que se sigue utilizando oficialmente la CIE-9 (aunque en el ámbito psiquiátrico se tienda a utilizar extraoficialmente la CIE-10) ${ }^{7}$.

El sistema de clasificación de Ibermutuamur durante el periodo de estudio y en el que están codificados los diagnósticos es la CIE 9 MC: ICD-9-CM (International Classification of Diseases, Ninth Revision, Clinical Modification).

Tanto para el DSM VI como para la CIE 10 el trastorno adaptativo es un fenómeno dependiente del estrés, que debe tener un origen identificable, pero que carece de una lista de síntomas. Ninguno de los síntomas es por sí solo de suficiente gravedad o importancia como para justificar un diagnóstico más específico.

La presencia de un agente estresante es esencial antes de poder hacer el diagnóstico de Trastorno de adaptación mientras los síntomas varíen e incluyan los que se encuentran en otros trastornos psiquiátricos comunes ${ }^{8}$.

El riesgo de aparición y la forma de expresión de las manifestaciones de los Trastornos de adaptación están determinados de un modo importante, más que en el caso de otros trastornos, por una predisposición o vulnerabilidad individual.

Si tenemos en cuenta las diferencias individuales de las capacidades de adaptación, ciertos factores de estrés pueden ser muy estresantes para un paciente y no serlo en absoluto para otro. Sin pautas cuantificables para medir los factores de estrés, el diagnóstico es a menudo determinado por la naturaleza de la respuesta individual.

Bawmweister y Kufner (2009) basándose en una revisión que destaca la falta de datos relativos a los Trastornos de adaptación, y que concluye que debería esperarse a disponer de datos suficientes antes de realizar este diagnóstico, refieren que muchos pacientes que no cumplen criterios diagnósticos más específicos, pero que siguen considerándose en la necesidad de recibir tratamiento, son diagnosticados de Trastorno de adaptación como si de un diagnóstico "comodín" se tratara. Esto conduce a una incorrecta evaluación de esta patología, que tiene como resultado una importante proporción de pacientes recibiendo tratamientos que pueden no ser adecuados. Consideran necesaria una revisión de los Trastornos de adaptación y de esta manera, establecer una frontera que delimite qué diferencia y permite distinguir entre Trastorno de adaptación, el proceso humano normal de adaptación y otros trastornos más específicos.

Todas estas consideraciones ponen de manifiesto la necesidad de evaluar correctamente los Trastornos de adaptación. Un correcto diagnóstico y tratamiento proporcionan la posibilidad de ajustar el tiempo de incapacidad laboral de esta patología al necesario para conseguir su correcta resolución. No hay que olvidar que, junto a los episodios depresivos y los trastornos mixtos ansioso-depresivos, han sido señalados como una de las causas más importantes de trastorno mental.

Es por ello que he considerado importante revisar la evolución de esta patología en la Mutua, con el objetivo de tener datos e información que puedan permitirnos mejorar su gestión y ajustar la duración de los procesos, de forma que los beneficios conseguidos repercutan en el paciente, la Mutua, la empresa y la sociedad en general.

Para cumplir este objetivo me planteo una revisión del comportamiento de la Incapacidad Temporal por Contingencias Comunes en los trabajadores con el diagnostico de Trastorno de adaptación controlados por Ibermutuamur en los años 2008, 2009 y 2010, realizar el análisis epidemiológico de estos procesos y valorar, en relación con los resultados obtenidos, si existe alguna variable que pueda determinar la toma de decisiones en la gestión de los procesos por contingencias comunes para acortar los periodos de incapacidad laboral. 


\section{MATERIAL Y MÉTODOS}

Para el análisis de resultados se ha realizado un estudio epidemiológico observacional retrospectivo descriptivo con componentes analíticos en una serie de casos.

Se ha utilizado para el tratamiento estadístico de la información el paquete Statistical Package for the Social Sciences (SPSS) versión 17.0.

Los datos se han obtenido del programa e-sanit@rio+, que contiene toda la información relativa a los trabajadores con procesos en baja de Ibermutuamur, Mutua de Accidentes de Trabajo y Enfermedades Profesionales de la Seguridad Social registrada en España con el número 274. Se han seleccionado todos los procesos con baja controlada por incapacidad laboral de contingencias comunes en todo el territorio nacional durante los años 2008, 2009 y 2010 con el diagnóstico CIE 9 - MC 309 Reacción de Adaptación.

De la base de datos excel inicial resultante, se seleccionan los datos referentes a: historia clínica (variable principal de registro de procesos), edad, sexo, fecha de la baja médica, fecha del alta médica, duración del proceso en días, recaídas, provincia en la que se realiza el control del proceso, ocupación laboral del trabajador, seguimiento por médico de control y/o salud mental (psiquiatra o psicólogo), solicitud de propuesta de alta, base reguladora de la prestación, pago por cuenta propia o ajena, modo de pago directo o delegado y alta por incapacidad permanente.

De esta nueva base de datos se eliminan los registros de pago delegado de duración inferior a 15 días, ya que es a partir del decimosexto cuando la Mutua asume la prestación económica de la incapacidad laboral y a partir del cual realiza el control y seguimiento del proceso (Real decreto 575/1997 de 18 de abril).

La base de datos depurada y validada definitiva consta de $\mathbf{5 . 9 9 7}$ registros.

El periodo de estudio comprende los años 2008, 2009 y 2010, seleccionando los procesos de incapacidad laboral controlados en estos tres años, por lo que se incluyen los que se iniciaron en 2007 y continúan en 2008, y los que se inician en 2010 y no son dados de alta hasta 2011.

El seguimiento y control de los procesos de incapacidad en contingencias comunes se realiza, generalmente por médicos, especialistas en Medicina Familiar y Comunitaria la mayoría de ellos, a quienes nos referiremos en adelante como médicos de control. No obstante, estructuralmente, Ibermutuamur cuenta en su plantilla con otros especialistas que se encargan del control y seguimiento de los trabajadores diagnosticados con procesos de su especialidad. En este sentido, durante este periodo de tiempo, cuatro psiquiatras y 15 psicólogos en 2008, 11 en 2009 y 11 en 2010, compartían la gestión de los procesos de salud mental con los médicos de control.

Los diagnósticos están codificados utilizando la CIE 9 - MC para el código 309 Reacción de adaptación, vigente en el Servicio Público de Salud y en nuestro propio sistema informático en esas fechas.

Para la codificación de los datos laborales de los trabajadores se ha utilizado la Clasificación Nacional de Ocupaciones 1994 (CON-94). La Clasificación nacional de Ocupaciones 2011 (CON -11) se aprueba en el Real Decreto1591/2010 de 26 de diciembre.

Las provincias se codifican utilizando como fuente el código del instituto Nacional de Estadística.

Para su análisis, las bases reguladoras de la prestación económica de los trabajadores se han agrupado en tres tramos; hasta $30 €$, de 31 a $65 €$ y mayor a $65 €$. Se ha elegido un tramo medio que engloba la media de la base reguladora de los trabajadores de la Mutua en estos tres años.

La base reguladora media en el año 2008 fue de 44,37 €, la del año 2009 de 45,68 y la del año 2010 de 51,48 €. La diferencia entre las de los dos primeros años y el tercero 
se debe a un error en la grabación de la misma en algunos procesos, atribuyéndole un número fijo cuando todavía no se disponía del dato real y no corregirse posteriormente, lo que ha supuesto una disminución de la media.

Las propuestas de alta no se codifican, están registradas por la fecha de petición, y ese es el dato que se utiliza. Para el cálculo de los días transcurridos desde la baja médica de los procesos hasta la solicitud de la propuesta de alta se excluyen los procesos con recaídas para evitar errores en el cálculo.

\section{RESULTADOS}

El número total de procesos de trabajadores en incapacidad temporal por contingencias comunes con el diagnóstico de Trastorno de adaptación durante los años 2008, 2009 y 2010 en todo el territorio nacional han sido 5997.

El número total de procesos controlados en Ibermutuamur durante estos tres años fue de 275.638; $104.975(38,08 \%)$ en $2008,86.530(31,39 \%)$ en 2009 y 84.163 (30,53\%) en 2010.

De ellos, $31.886(11,57 \%)$ correspondieron a procesos controlados con patología psiquiátrica; 11.275 (10,74\%) en $2008,10.725$ (12,39\%) en 2009 y $9.889(11,75 \%)$ en 2010. Anexo 1

- $\quad$ Respecto al sexo, 3698 (61,7\%) son mujeres y 2299 (38,3\%) hombres.

\section{- Edad}

Se registran trabajadores con el diagnóstico seleccionado en todas las edades, desde los 18 a los 70 años.

El mayor número de procesos se registra a la edad de 35 años; 267 que suponen un $4.5 \%$ del total. Le siguen los trabajadores de 37 años; 243 (4.1\%), y 38 años; 230 (3,8\%).

Gráfico 1. Distribución por edad

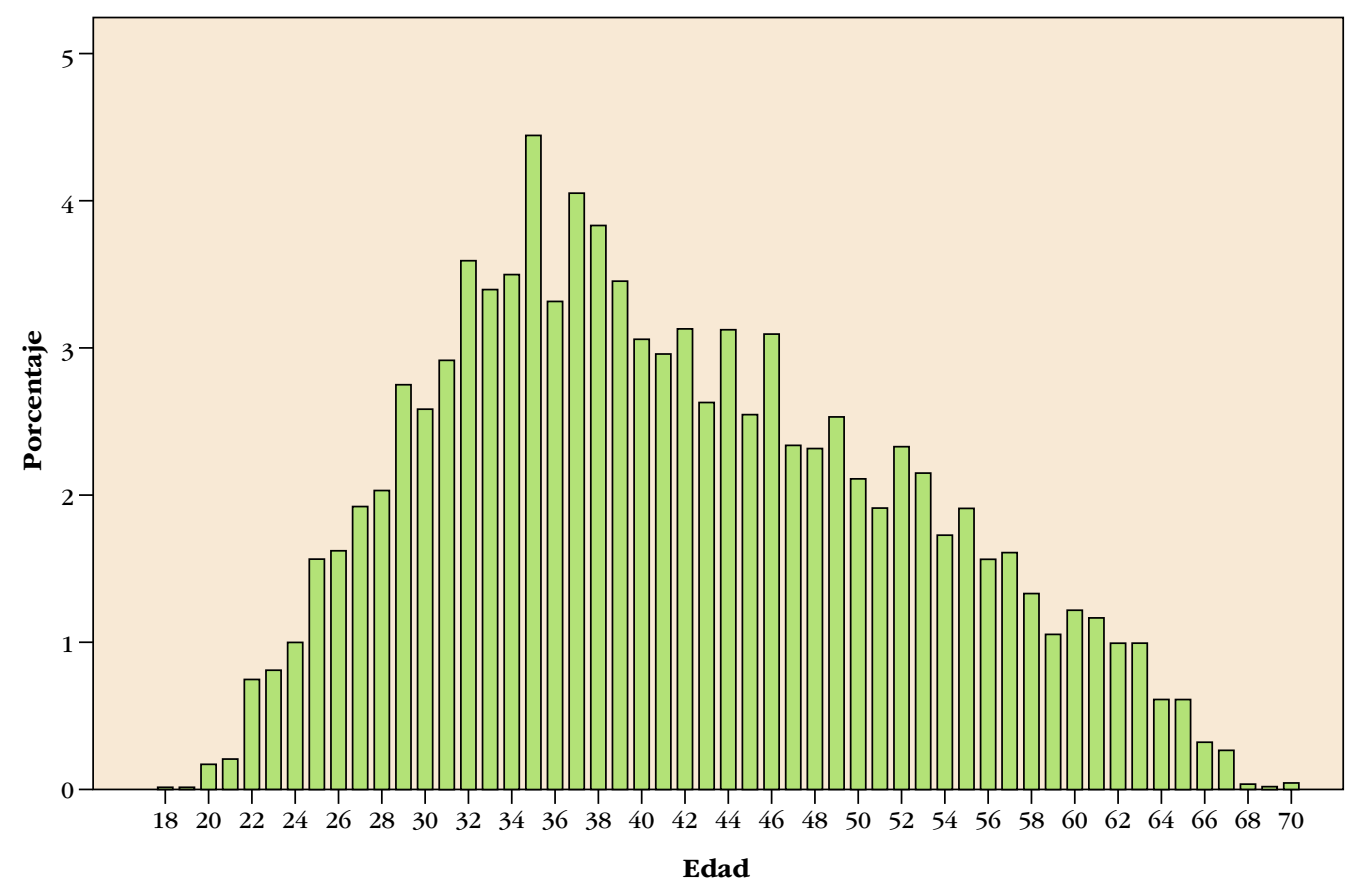


La distribución por tramos de edad ha sido:

- Hasta los 24 años: 179 (2,98\%).

- De los 25 a los 34 años: 1.553 (25,89\%).

— De los 35 a los 44 años: 2.040 (34,01\%).

- De los 45 a los 54 años: 1.264 (21,07\%).

- De los 55 a los 64 años: 755 (12,58\%).

— De 65 a 70 años: 80 (1,33\%).

- En cuanto a la actividad de los trabajadores solo se encuentra detallada para 5.354 $(89,27 \%)$.

El $17,7 \%$ de los trabajadores (1.060) pertenecen a la ocupación de Técnicos y profesionales de apoyo.

El 16,1 \% (963) se encuentran en Servicios de restauración, personales, protección y vendedores.

Otro 15\% (899) son Trabajadores no cualificados.

Entre los tres grupos suman el 48,72\% del total de los trabajadores estudiados.

El resto se distribuye profesionalmente como indica la tabla siguiente:

Gráfico 2. Distribución de los trabajadores en relación con el código de actividad

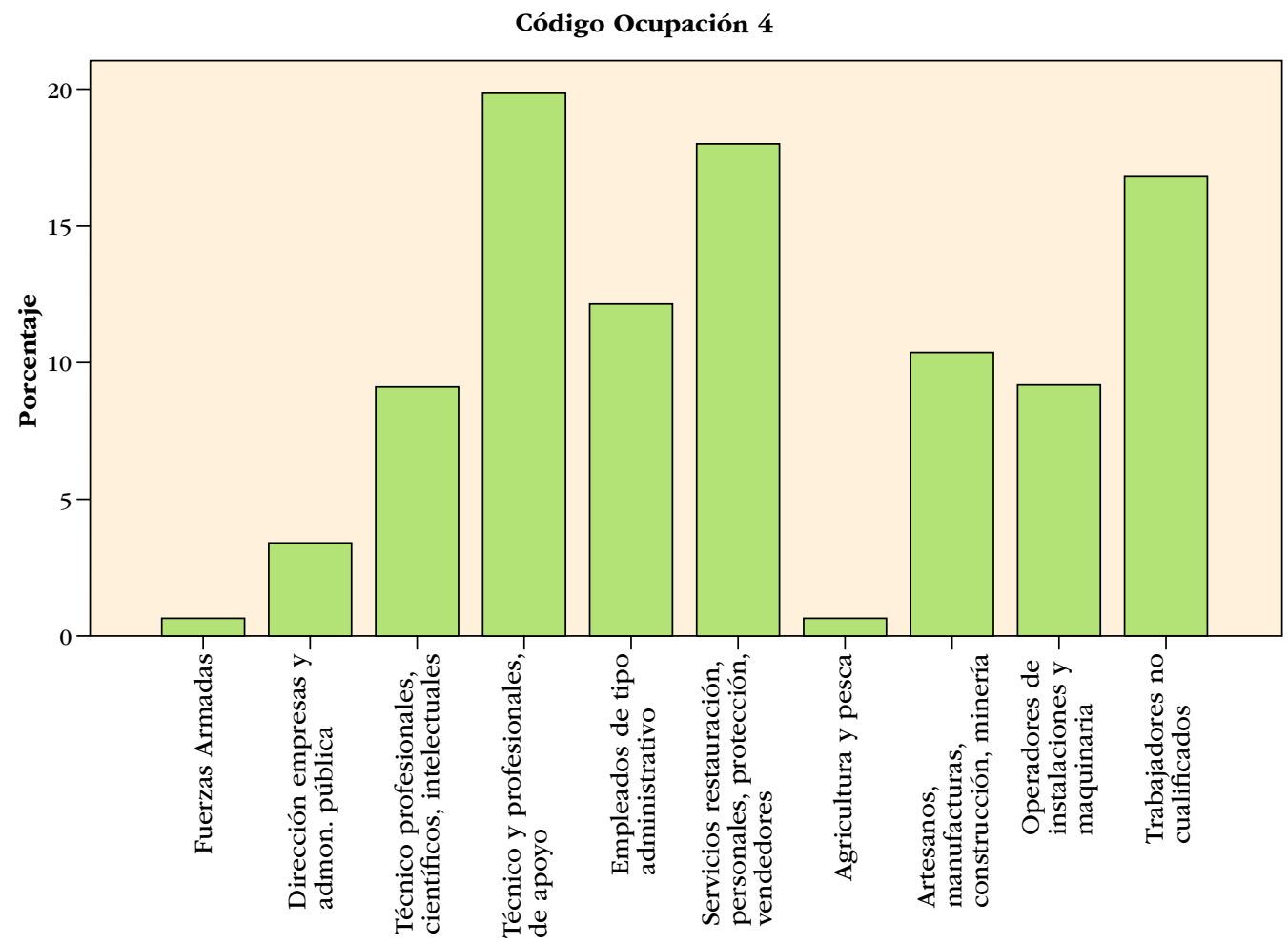




\section{- Provincia}

La distribución de los procesos por provincia es la siguiente:

Tabla I. Distribución de procesos por provincio

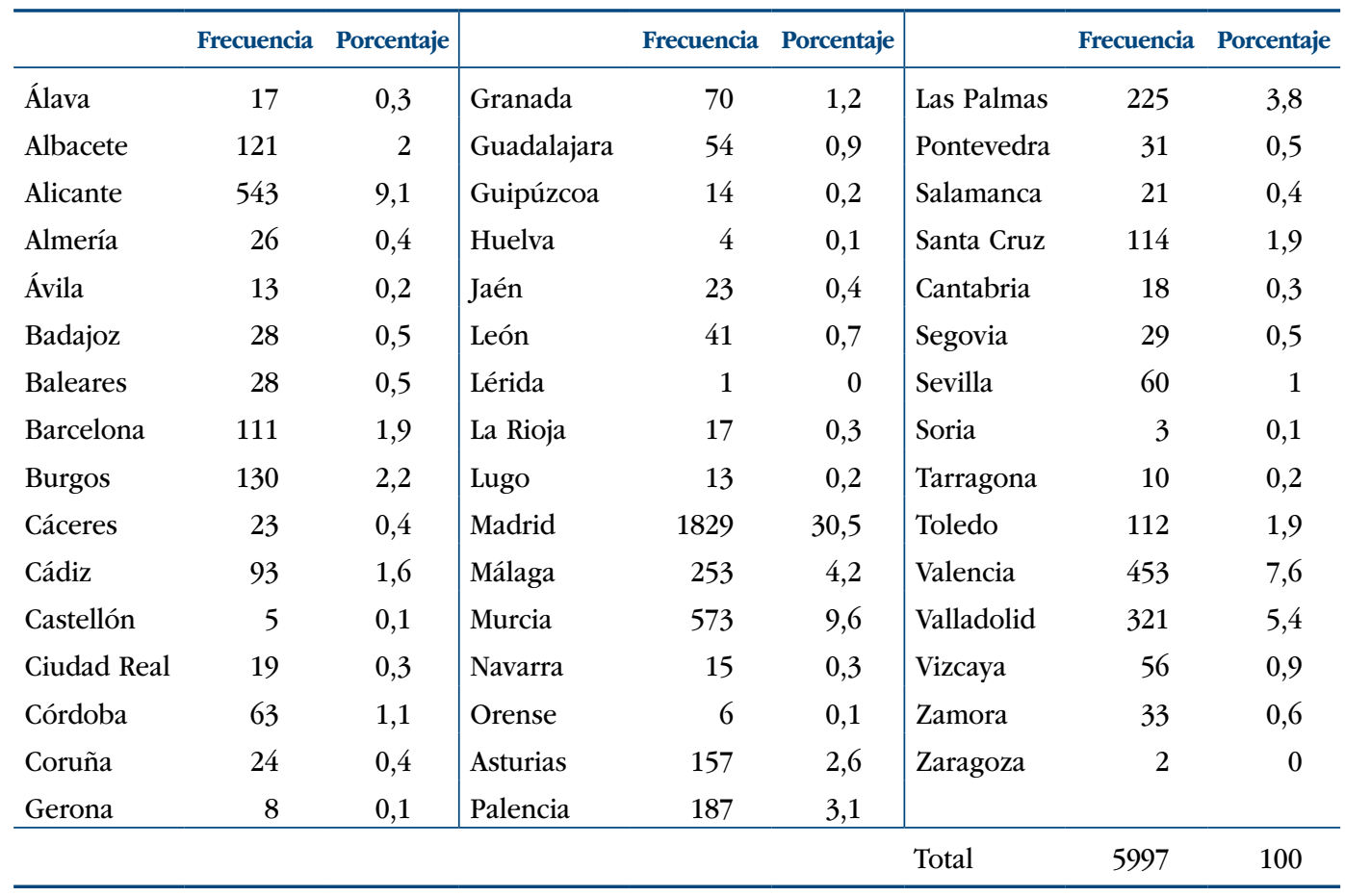

Tabla II. Proporción de procesos con patología psiquiátrica con relación al total de procesos por provincia

\begin{tabular}{lc|ll}
\hline $\begin{array}{c}\text { Mayor número de procesos } \\
\text { con patología psiquiátrica } \\
\text { con relación al número total } \\
\text { de procesos controlados }\end{array}$ & $\%$ & $\begin{array}{l}\text { Menor número de procesos } \\
\text { con patología psiquiátrica } \\
\text { con relación al número total } \\
\text { de procesos controlados }\end{array}$ & $\%$ \\
\hline Barcelona & 20,65 & La Rioja & 6,68 \\
Zaragoza & 19,89 & Segovia & 6,32 \\
Las Palmas de G. C. & 17,56 & Guadalajara & 6,02 \\
Valencia & 16,75 & Soria & 4,95 \\
Baleares & 14,69 & & \\
\hline
\end{tabular}

Tabla III. Proporción de procesos con diagnóstico. Reacción de adaptación con relación al número de procesos u por provincia

\begin{tabular}{lc|lc}
\hline $\begin{array}{l}\text { Mayor número de procesos } \\
\text { con diagnóstico R.A. con } \\
\text { relación al número de } \\
\text { procesos psiquiátricos }\end{array}$ & $\%$ & $\begin{array}{l}\text { Mayor número de procesos } \\
\text { con diagnóstico R.A. con } \\
\text { relación al número de } \\
\text { procesos psiquiátricos }\end{array}$ & $\%$ \\
\hline Ávila & 76,47 & Granada & 6,46 \\
Sta. Cruz de Tenerife & 49,57 & Sevilla & 6,27 \\
Las Palmas de G.C. & 41,51 & Zaragoza & 5,56 \\
Palencia & 39,62 & Pontevedra & 5,38 \\
Albacete & 36,78 & La Coruña & 5,36 \\
Burgos & 35,62 & Almería & 4,99 \\
& & Salamanca & 4,87 \\
& & Orense & 3,70 \\
& & Huelva & 2,30 \\
\hline
\end{tabular}


Madrid, Murcia y Alicante son las tres provincias con mayor número de trabajadores afectados por esta patología; 2945 trabajadores (49,10\%). Pero también son las tres provincias que reúnen el mayor número de trabajadores en situación de incapacidad laboral y los diagnosticados de patología psiquiátrica.

Solo se ve relación entre el porcentaje de procesos diagnosticados como patología psiquiátrica y el diagnóstico de Trastorno de adaptación en Las Palmas de Gran Canaria. En Canarias es donde se diagnostica con más frecuencia.

- Teniendo en cuenta el diagnóstico, comprobamos que el mayor número de trabajadores han sido diagnosticado con la descripción genérica para el código 309

- Reacción de adaptación: 2.638 procesos (44,0\%).

Le siguen en diagnóstico los códigos 309.28 Reacción de adaptación con características emocionales mixtas: 1.281 procesos $(21,4 \%)$ y 309.24 Reacción de adaptación con humor de ansiedad: 926 procesos (15,4\%). Entre los dos representan el 36,80\% del total.

Tabla IV. Distribución de procesos por código diagnóstico y tramo de edad

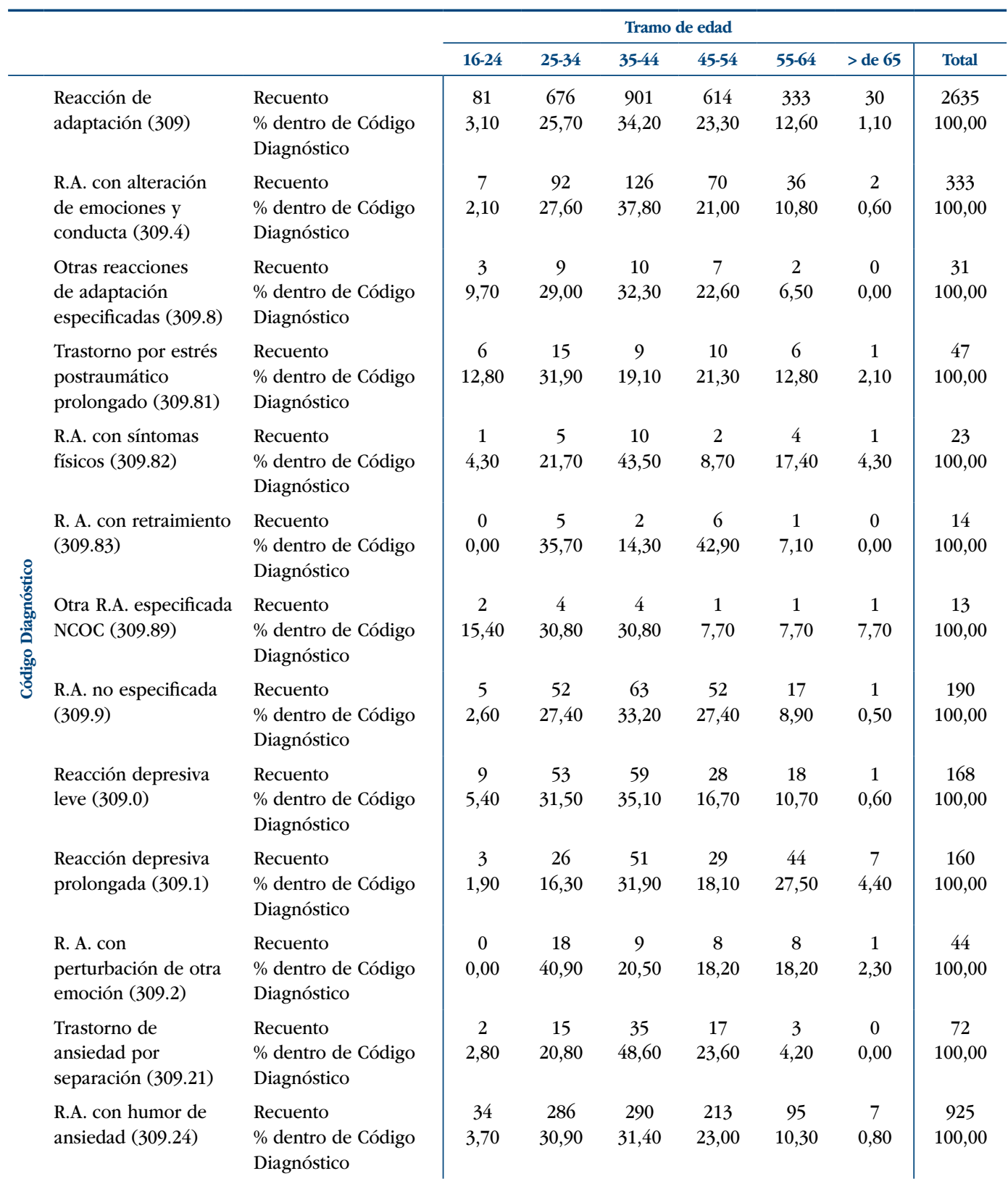




\begin{tabular}{|c|c|c|c|c|c|c|c|c|c|}
\hline & & & \multicolumn{7}{|c|}{ Tramo de edad } \\
\hline & & & $16-24$ & $25-34$ & $35-44$ & $45-54$ & $55-64$ & $>$ de 65 & Total \\
\hline \multirow{8}{*}{ 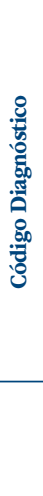 } & R. A. con & Recuento & 24 & 281 & 456 & 314 & 179 & 27 & 1281 \\
\hline & $\begin{array}{l}\text { características } \\
\text { emocionales mixtas } \\
(309.28)\end{array}$ & $\begin{array}{l}\text { \% dentro de Código } \\
\text { Diagnóstico }\end{array}$ & 1,90 & 21,90 & 35,60 & 24,50 & 14,00 & 2,10 & 100,00 \\
\hline & Otra R.A. con & Recuento & 1 & 1 & 3 & 4 & 1 & 0 & 10 \\
\hline & $\begin{array}{l}\text { perturbación de otras } \\
\text { emociones }(309.29)\end{array}$ & $\begin{array}{l}\text { \% dentro de Código } \\
\text { Diagnóstico }\end{array}$ & 10,00 & 10,00 & 30,00 & 40,00 & 10,00 & 0,00 & 100,00 \\
\hline & R.A. con alteración & Recuento & 1 & 15 & 12 & 9 & 7 & 1 & 45 \\
\hline & $\begin{array}{l}\text { predominante de } \\
\text { conducta }(303.3)\end{array}$ & $\begin{array}{l}\text { \% dentro de Código } \\
\text { Diagnóstico }\end{array}$ & 2,20 & 33,30 & 26,70 & 20,00 & 15,60 & 2,20 & 100,00 \\
\hline & \multirow[t]{2}{*}{ Total } & Recuento & 179 & 1553 & 2040 & 1384 & 755 & 80 & 5991 \\
\hline & & $\begin{array}{l}\text { \% dentro de Código } \\
\text { Diagnóstico }\end{array}$ & 3,00 & 25,90 & 34,10 & 23,10 & 12,60 & 1,30 & 100,00 \\
\hline
\end{tabular}

Después de realizar los contrastes de hipótesis (test ji-cuadrado de Pearson) para comparación de porcentajes entre variables obtenemos dependencias entre las variables diagnóstico y edad $(\mathrm{p}<0,05)$.

El diagnóstico genérico de Reacción de adaptación se presenta con mayor frecuencia en los tramos de edad comprendidos entre los 35 y 44 años: 901, 15,03\% del total de registros, y los 45 y 54 años: 614 procesos, 10,24\%.

Inmediatamente posterior, el diagnóstico de R. A. con características emocionales mixtas es el más frecuente para los mismos tramos de edad d: $452(7,54 \%)$ y $314(5,24 \%)$ procesos respectivamente.

Realmente son los diagnósticos más frecuentes para cualquier tramo de edad, excepto entre los 16 y 34 años, en el que es más frecuente el de R. A. con humor de ansiedad (309.24).

El tramo de edad comprendido entre los 35 y 44 años, es el de mayor frecuencia de diagnóstico para casi todos los subtipos diagnósticos.

\section{- Seguimiento y control de los procesos}

Los médicos de control siguieron 4.911 procesos (81,9\% del total), 1.006 procesos los psicólogos (16,8\%) y 80 los psiquiatras (1,3\%).

El código 309 Reacción de adaptación es el más frecuentemente diagnosticado por los tres: 1990 (40,52\%) médicos, 639 (63,51\%) psicólogos y 29 (36,25\%) psiquiatras.

Le sigue el código 309.28 R. A. con características emocionales mixtas para médicos de control y psicólogos, 1084 (22,07\%) y $173(17,19 \%)$ respectivamente y el código 309.24 R.A. con humor de ansiedad en el caso de los psiquiatras, 24 (30\%), código que también es el tercero en frecuencia para médicos de control y psicólogos: $809(16,47 \%)$ y 109 $(10,83)$ procesos.

Los psicólogos son los que mayor número de procesos mantienen en el código genérico 309.

La duración media de los procesos controlados por un médico de control fue de 162 días, la de los procesos controlados por un psiquiatra 246 días y 168 días si un psicólogo realizó el control y seguimiento de los mismos.

- El 90,3\% de todos los trabajadores (5.416) no experimentó recaída en su proceso.

Tuvieron recaída 581. El 54,38\% de las recaídas (316) se producen en mujeres.

El 30,63\% de las recaídas se reparten a partes iguales entre las ocupaciones de Técnicos y profesionales de apoyo y Trabajadores no cualificados. Si le añadimos el grupo 
de "Trabajadores de los servicios de restauración, personales, proyección y vendedores de los comercios" el porcentaje se eleva al $44 \%$.

El 35,97\% se producen en el tramo de edad comprendido entre los 35 y 44 años.

El mayor porcentaje de recaídas recae en el código genérico 309 (42,68\%), pero el 26,85 del total se corresponden con el código diagnóstico 309.28 "R.A. con características emocionales mixtas (con una diferencia mayor del 50\% con el siguiente 309.24 R.A. con humor de ansiedad). Estos resultados coinciden con los códigos diagnósticos más frecuentes.

La provincia que mayor número de recaídas presenta, con relación al número total de procesos en ella diagnosticados, es Madrid 246 (13,44\%). Le siguen Las Palmas de G. C. con el $12,98 \%$ y Valencia con el $10,15 \%$.

- Respecto al pago de la prestación al trabajador, el 66,1\% (3.965) son trabajadores en pago delegado y 33\% (2.032) en pago directo, de los que el 8,6\% (516) son de cuenta propia y el 25,3\% (1.516) de cuenta ajena.

Total de trabajadores por cuenta ajena: $5.481(91,45)$.

Gráfico 3. Distribución por modo de pago.

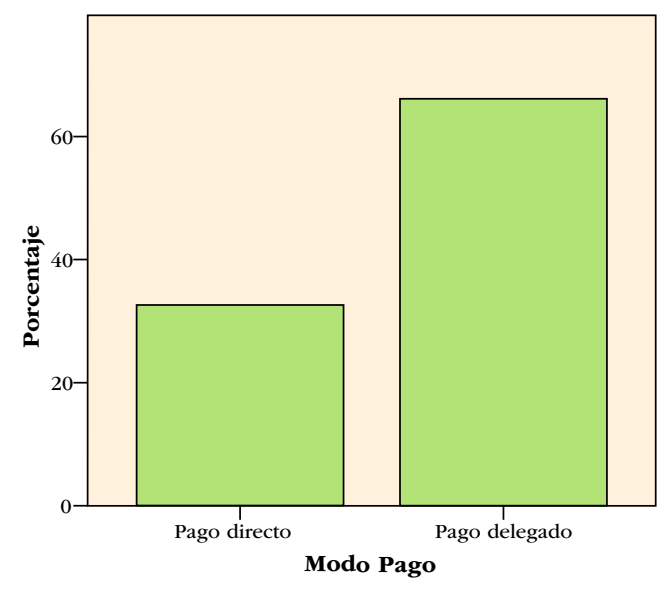

Gráfico 4. Distribución cuenta propia/cuenta ajena

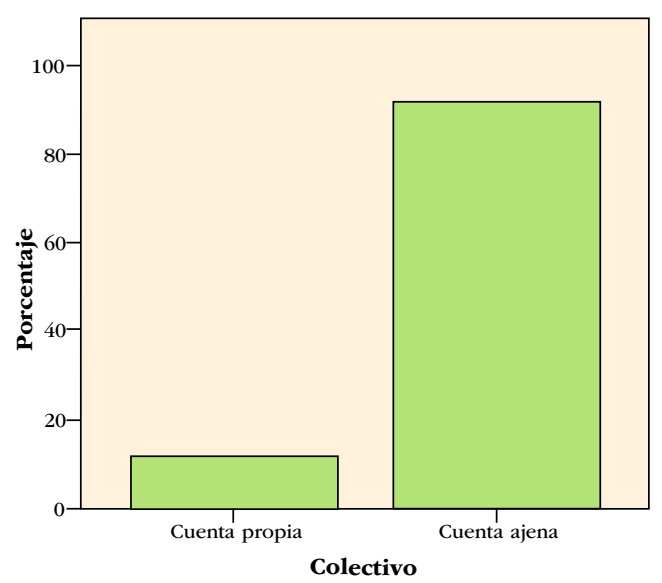

\section{- Base reguladora}

El mayor porcentaje de trabajadores tienen una base reguladora comprendida entre 31 y $65 €$, tramo en el que se encuentra la media de la base reguladora de los trabajadores de la Mutua en ese periodo.

Los procesos de trabajadores con base reguladora inferior a $31 €$ tuvieron un duración media de 179 días, 161 días los de base reguladora entre 31 y $65 €$ y 156 días los de base reguladora mayor de $65 €$. 
Gráfico 5. Porcentaje de trabajadores en relación con la base reguladora de su prestación

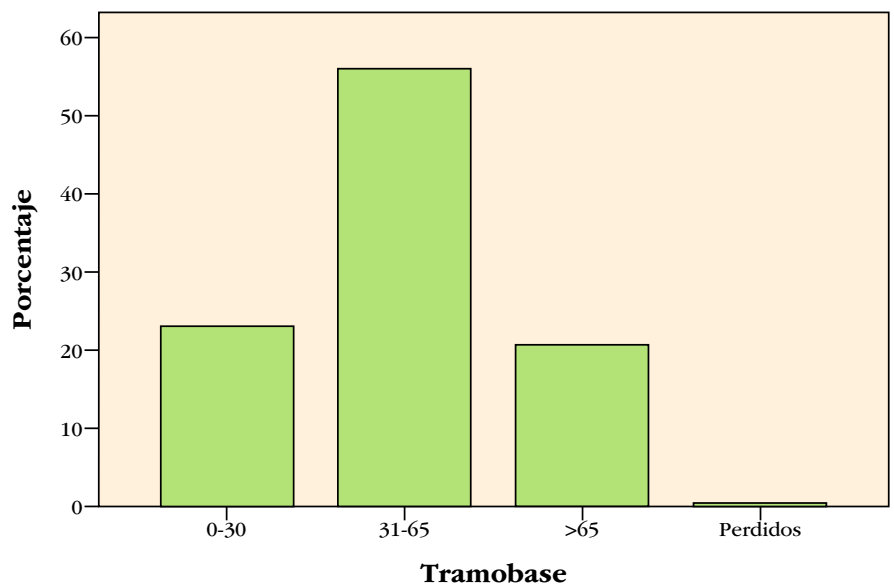

- Los datos del año de baja y alta de los procesos son:

Tabla V. Año de baja y alta de los procesos

\begin{tabular}{lccc|cccc}
\hline & \multicolumn{3}{c|}{ Año de baja } & \multicolumn{3}{c}{ Año de alta } \\
\cline { 2 - 7 } & Frecuencia & Porcentaje & $\begin{array}{c}\text { Porcentaje } \\
\text { válido }\end{array}$ & & Frecuencia & Porcentaje & $\begin{array}{c}\text { Porcentaje } \\
\text { válido }\end{array}$ \\
\hline $\mathbf{2 0 0 7}$ & 485 & 8,1 & 8,1 & & & & \\
$\mathbf{2 0 0 8}$ & 2274 & 37,9 & 37,9 & $\mathbf{2 0 0 8}$ & 1958 & 32,6 & 33,6 \\
$\mathbf{2 0 0 9}$ & 1881 & 31,4 & 31,4 & $\mathbf{2 0 0 9}$ & 2002 & 33,4 & 34,4 \\
$\mathbf{2 0 1 0}$ & 1357 & 22,6 & 22,6 & $\mathbf{2 0 1 0}$ & 1629 & 27,2 & 28 \\
Total & 5997 & 100 & 100 & $\mathbf{2 0 1 1}$ & 230 & 3,8 & 4 \\
& & & & Total & 5819 & 97 & 100 \\
& & & & Perdido & 178 & 3 & \\
& & & & Total & 5997 & 100 & \\
\hline
\end{tabular}

- En cuanto a los meses de alta y baja de los procesos no se observa ninguna diferencia importante que indique alguna tendencia en este sentido.

Tabla VI. Meses de baja y alta de los procesos

\begin{tabular}{lcc|lcc}
\hline & Mes de baja & & & \multicolumn{2}{c}{ Mes de alta } \\
\cline { 2 - 3 } Enero & Frecuencia & Porcentaje & & Frecuencia & Porcentaje \\
\cline { 2 - 3 } Octubre & 463 & 7,7 & Enero & 612 & 10,2 \\
Noviembre & 567 & 9,5 & Octubre & 528 & 8,8 \\
Diciembre & 480 & 8 & Noviembre & 482 & 8 \\
Febrero & 371 & 6,2 & Diciembre & 428 & 7,1 \\
Marzo & 470 & 7,8 & Febrero & 427 & 7,1 \\
Abril & 495 & 8,3 & Marzo & 532 & 8,9 \\
Mayo & 531 & 8,9 & Abril & 500 & 8,3 \\
Junio & 556 & 9,3 & Mayo & 518 & 8,6 \\
Julio & 570 & 9,5 & Junio & 547 & 9,1 \\
Agosto & 538 & 9 & Julio & 536 & 8,9 \\
Septiembre & 362 & 6 & Agosto & 405 & 6,8 \\
Total & 594 & 9,9 & Septiembre & 482 & 8 \\
\hline
\end{tabular}


- Distribución de los procesos según los días en IT.

Gráfico 6. Distribución de los procesos en relación con los días de baja laboral

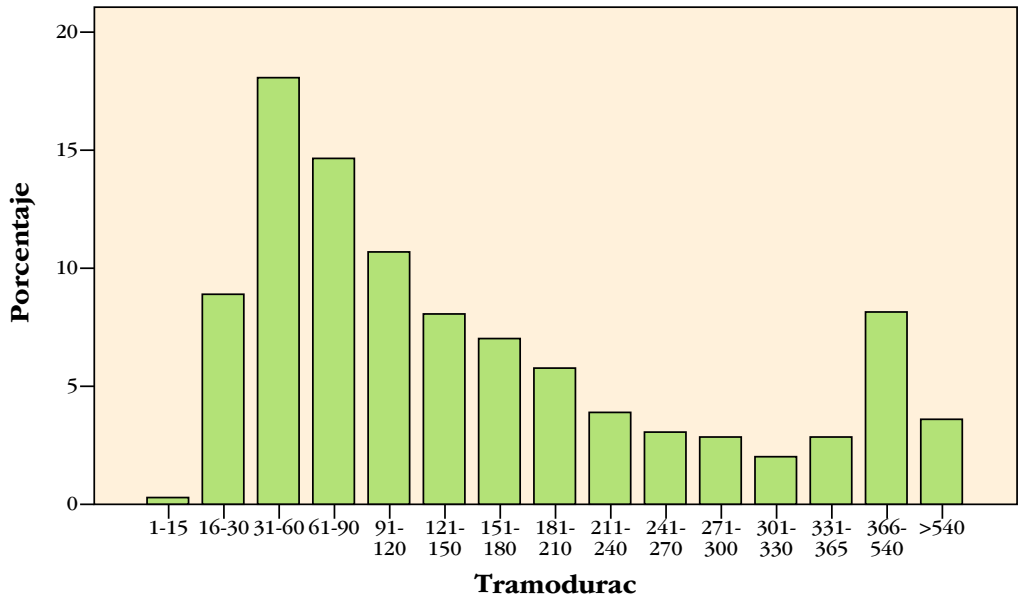

El 18,2\% del total de los procesos (1.089) tiene una duración entre 31 y 60 días.

El 52,6\% de las bajas (3.153) se concentran en duraciones inferiores a cuatro meses.

El 11,8\% (705) supera el año en situación de incapacidad laboral y el 3,6\% (214) los 18 meses.

Después de realizar los contrastes de hipótesis (test ji-cuadrado de Pearson) para comparación de porcentajes entre las variables duración y edad obtenemos dependencias entre las variables $(\mathrm{p}<0,05)$.

El mayor número de procesos está en el tramo de duración de 31 a 60 días para las edades comprendidas entre los 35 y los 44 años, 357 (5,95\%) y entre los 25 y 34 años, 337 (5,61\%).

Este tramo de duración se repite para cualquier tramo de edad excepto para mayores de 65 años.

Tabla VII. Duración de los procesos en relación con la edad del trabajador

\begin{tabular}{|c|c|c|c|c|c|c|c|c|}
\hline & \multicolumn{7}{|c|}{ Tramedad } \\
\hline & & $16-24$ & 25-34 & $35-44$ & $45-54$ & 55-64 & $>\operatorname{de} 65$ & Total \\
\hline \multirow{17}{*}{ 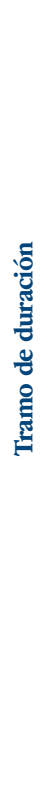 } & $0-15$ & 0 & 8 & 1 & 7 & 1 & 0 & 17 \\
\hline & $16-30$ & 20 & 174 & 173 & 95 & 63 & 5 & 530 \\
\hline & $31-60$ & 44 & 337 & 357 & 219 & 126 & 6 & 1089 \\
\hline & $61-90$ & 32 & 239 & 300 & 224 & 72 & 7 & 874 \\
\hline & 91-120 & 26 & 179 & 237 & 129 & 64 & 4 & 639 \\
\hline & $121-150$ & 14 & 144 & 165 & 96 & 60 & 4 & 483 \\
\hline & 151-180 & 8 & 101 & 143 & 106 & 58 & 8 & 424 \\
\hline & 181-210 & 4 & 86 & 118 & 78 & 51 & 9 & 346 \\
\hline & 211-240 & 4 & 55 & 84 & 55 & 37 & 1 & 236 \\
\hline & 241-270 & 4 & 36 & 66 & 56 & 19 & 5 & 186 \\
\hline & 271-300 & 4 & 37 & 58 & 33 & 37 & 4 & 173 \\
\hline & $301-330$ & 5 & 19 & 41 & 36 & 14 & 2 & 117 \\
\hline & $331-365$ & 4 & 29 & 54 & 50 & 30 & 5 & 172 \\
\hline & $366-540$ & 7 & 76 & 176 & 135 & 79 & 18 & 491 \\
\hline & $>540$ & 3 & 33 & 67 & 65 & 44 & 2 & 214 \\
\hline & \multirow[t]{2}{*}{ Total } & 179 & 1553 & 2040 & 1384 & 755 & 80 & 5991 \\
\hline & & $3,00 \%$ & $25,90 \%$ & $34,10 \%$ & $23,10 \%$ & $12,60 \%$ & $1,30 \%$ & $100,00 \%$ \\
\hline
\end{tabular}


Gráfico 7. Duración de los procesos en relación con la edad del trabajador

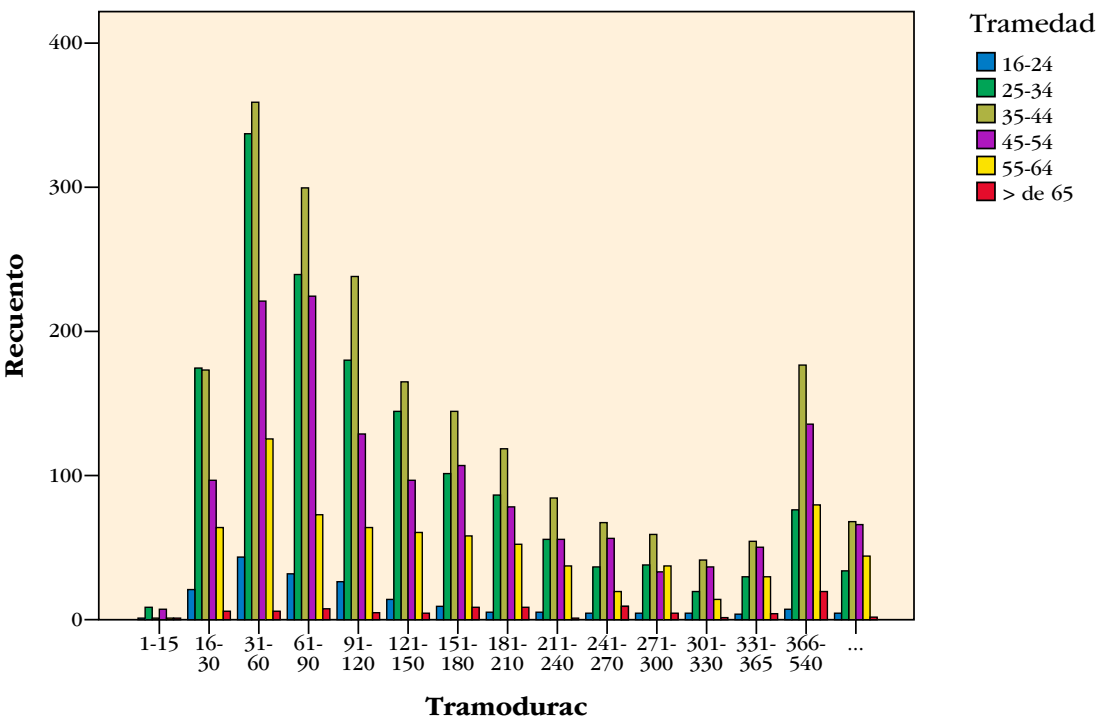

La mayor duración media de la baja médica se produce para el diagnóstico R.A. con síntomas físicos (309.82) que no está reflejado en los Tiempos Estándar de Incapacidad temporal del Instituto nacional de la Seguridad Social (INSS) ${ }^{9}$.

Los procesos con mayor duración media de la baja no son los procesos diagnosticados con mayor frecuencia.

Si comparamos la duración media de las bajas de cada diagnóstico con el Tiempo Estándar del INSS, vemos que lo supera con un amplísimo margen (Tabla VIII).

La duración del 25\% de los procesos (percentil 25) aunque por encima del Tiempo estándar del INSS no muestra cifras tan alejadas del mismo como la duración media, a excepción del diagnóstico 309.81 Trastorno por estrés postraumático prolongado, en que incluso está por debajo.

No obstante, en comparación con la mediana (percentil 50) duración del 50\% de los procesos, la gran diferencia para la mayoría de los diagnósticos es todavía más manifiesta, incluyendo al diagnóstico 309.81 .

Tabla VIII. Duración de los procesos por diagnóstico con relación a los Tiempos Estándar del INSS

\begin{tabular}{llcccc}
\hline & \multicolumn{1}{c}{ Código diagnóstico CIE 9 MC } & $\begin{array}{c}\text { Duración } \\
\text { media de la } \\
\text { baja en días }\end{array}$ & $\begin{array}{c}\text { Días en baja } \\
\text { (Percentil 25) }\end{array}$ & $\begin{array}{c}\text { Días en baja } \\
\text { (Percentil 50) }\end{array}$ & $\begin{array}{c}\text { Tiempo es- } \\
\text { tándar INSS } \\
\text { en días }\end{array}$ \\
\hline $\mathbf{3 0 9}$ & Reacción de adaptación & 151 & 55 & 108 & 20 \\
$\mathbf{3 0 9 , 0}$ & Reacción depresiva leve & 106 & 35 & 73 & 30 \\
$\mathbf{3 0 9 , 1}$ & Reacción depresiva prolongada & 231 & 94,75 & 197,5 & 60 \\
$\mathbf{3 0 9 , 2}$ & R.A. con perturbación de otra emoción & 111 & 46,5 & 76 & 20 \\
$\mathbf{3 0 9 , 2 1}$ & Trastorno de ansiedad por separación & 134 & 42,5 & 83 & 14 \\
$\mathbf{3 0 9 , 2 4}$ & R.A. con humor de ansiedad & 157 & 53 & 100 & 14 \\
$\mathbf{3 0 9 , 2 8}$ & R.A. con características emocionales mixtas & 193 & 71 & 148 & 30 \\
$\mathbf{3 0 9 , 2 9}$ & Otra R.A. con perturbación de otras emociones & 270 & 144 & 263 & 20 \\
$\mathbf{3 0 9 , 3}$ & R.A. con alteración predominante de conducta & 196 & 61 & 142 & 20 \\
$\mathbf{3 0 9 , 4}$ & R.A. con alteración de emociones y conducta & 181 & 65 & 119 & 20 \\
$\mathbf{3 0 9 , 8}$ & Otras reacciones de adaptación especificadas & 162 & 51,5 & 105 & 20 \\
$\mathbf{3 0 9 , 8 1}$ & Trastorno por estrés postraumático prolongado & 265 & 66,75 & 235,5 & 90 \\
$\mathbf{3 0 9 , 8 2}$ & R.A. con síntomas físicos & 273 & 73,5 & 181 & 66 \\
$\mathbf{3 0 9 , 8 3}$ & R.A. con retraimiento & 140 & 39,5 & 86 & 210 \\
$\mathbf{3 0 9 , 8 9}$ & Otra R.A. especificada NCOC & 209 & 124 & 39,25 & 69 \\
$\mathbf{3 0 9 , 9}$ & R.A. no especificada & & & 20 \\
\hline
\end{tabular}


La Official Disability Guideline ${ }^{10}$, elaborada por el Workloss Data Institute, entidad privada en EE.UU. desde 1996, y que evalúa los días estimados de ausencia al trabajo en el percentil 50 (mediana) como duración esperada y en el percentil 90 (a riego) como duración máxima, establece para estos diagnósticos los siguientes datos [sólo incluyendo los procesos de duración mayor de 7 días (claims data) y no los de duración menor (all absence)]:

Tabla IX. Duración de los procesos por diagnóstico con relación a la Official Disability Guideline

\begin{tabular}{|c|c|c|c|c|}
\hline & \multirow{2}{*}{ Código diagnóstico CIE 9 MC } & \multirow{2}{*}{$\begin{array}{l}\text { Días en baja } \\
\text { (Percentil 50) }\end{array}$} & \multicolumn{2}{|c|}{ Official Disability Guideline WDI } \\
\hline & & & Percentil 50 & Percentil 90 \\
\hline 309 & Reacción de adaptación & 108 & 16 & 45 \\
\hline 309,0 & Reacción depresiva leve & 73 & 53 & 136 \\
\hline 309,1 & Reacción depresiva prolongada & 197,5 & 48 & 69 \\
\hline 309,2 & R.A. con perturbación de otra emoción & 76 & 21 & 93 \\
\hline 309,21 & Trastorno de ansiedad por separación & 83 & 21 & 93 \\
\hline 309,24 & R.A. con humor de ansiedad & 100 & 21 & 93 \\
\hline 309,28 & R.A. con características emocionales mixtas & 148 & 69 & 127 \\
\hline 309,29 & Otra R.A. con perturbación de otras emociones & 263 & 21 & 93 \\
\hline 309,3 & R.A. con alteración predominante de conducta & 142 & 22 & 221 \\
\hline 309,4 & R.A. con alteración de emociones y conducta & 119 & 50 & 215 \\
\hline 309,8 & Otras reacciones de adaptación especificadas & 105 & 15 & 52 \\
\hline 309,81 & Trastorno por estrés postraumático prolongado & 235,5 & 39 & 75 \\
\hline 309,82 & R.A. con síntomas físicos & 181 & 15 & 52 \\
\hline 309,83 & R.A. con retraimiento & 66 & 15 & 52 \\
\hline 309,89 & Otra R.A. especificada NCOC & 210 & 15 & 52 \\
\hline 309,9 & R.A. no especificada & 69 & 15 & 29 \\
\hline
\end{tabular}

La duración de los procesos para el 50\% de nuestros trabajadores y para casi todos los diagnósticos, se aproxima más a la duración de los procesos para el 90\% de los trabajadores en la Guía americana, duración "a riesgo" o duración máxima. Constituyen la excepción los diagnósticos 309.0, 309.2, 309.3, 309.4 y 309.21, que aún alejándose mucho de la duración americana para el 50\% de los trabajadores, mantienen una importante duración a la baja con relación a su duración máxima.

Ibermutuamur, publica, en el año 2011, Duración de la Incapacidad Temporal asociada a diferentes patologías en trabajadores españoles ${ }^{3}$, investigación financiada mediante subvención recibida de acuerdo con lo previsto en la Orden TIN/1902/2009 de 10 de julio (premios para el Fomento de la Investigación de la Protección social -FIPROS-).

Para el estudio de los Trastornos de adaptación en C. Comunes calcula únicamente el estándar de Ibermutuamur (mediana de la duración) para los códigos 309 Reacción de adaptación siguientes:

Tabla X. Duración mediana de los procesos del estudio Ibermutuamur del año 2011

\begin{tabular}{lccccc}
\hline \multicolumn{7}{c}{ Datos Ibermutuamur años 2003 a 2007} \\
& 309 & 309.0 & 309.24 & 309.28 & 309.4 \\
\hline Percentil 50 (mediana) & 78 & 92 & 74 & 85 & 74 \\
\hline
\end{tabular}

Se observan importantes diferencias en cuanto a los datos ya que no son comparables debido a la diferencias en la selección y cálculo de la duración de los procesos. 


\section{- Propuestas de alta}

Se solicitó propuesta de alta en 1.520 del total de los procesos (25,34\%).

En el año 2008 se solicitó propuesta de alta para 568 procesos $(20,58 \%$ de los procesos controlados en ese año y un 37,36\% de las solicitadas en total en los tres años). De ellos a $76(13,3 \%)$ se les solicitó una segunda propuesta. Y para alguno de ellos incluso una cuarta y una quinta propuesta (con alta a los tres meses y medio).

La mayoría, se solicitó para el código diagnóstico genérico Reacción de Adaptación 309 (258), seguidas por el 309.28, R.A. con características emocionales mixtas con 118 y 309.24 R.A. con humor de ansiedad, 96.

En el año 2009 se solicitó propuesta de alta para 510 procesos $(27,11 \%$ de los procesos controlados ese año y un 33,55\% de las solicitadas en los tres años). De ellos a $51(10 \%)$ se les solicitó una segunda propuesta. E igualmente en este año, para algunos procesos hubo que solicitar una tercera propuesta llegando en un caso a la sexta solicitud (con alta a los tres meses y medio).

La mayoría 252 se solicitó para el código diagnóstico genérico 309, seguidas del 309.24 (105), y 309.28 (82).

En el año 2010 se solicitó propuesta de alta para 442 procesos $(32,57 \%$ de los procesos controlados ese año y un $29,07 \%$ de las solicitadas en los tres años). De ellos a $55(12,4 \%)$ se les solicitó una segunda propuesta, y para algunos una cuarta e incluso una quinta propuesta, consiguiendo el alta inmediata.

En relación con los diagnósticos de los procesos, la mayoría, 203 se solicitó para el código diagnóstico genérico 309, seguidas del 309.24 (79) y 309.28 (77).

La duración media de los procesos sin recaída ( 5416 procesos) cuando se solicita la propuesta de alta es de 173,41 días.

En el año 2008 fue de 126,98 días, en el año 2009 de 118,81 días y en el año 2010 de 78,16 días.

Tabla XI. Tiempo transcurrido desde la baja hasta la primera propuesta de alta

\begin{tabular}{cc}
\hline \multicolumn{2}{c}{$\begin{array}{c}\text { Tiempo desde baja hasta primera propuesta de alta } \\
\text { (sólo episodios sin recaida) }\end{array}$} \\
\hline Percentil 25 & 55,00 \\
Percentil 50 & 95,00 \\
Percentil 75 & 155,00 \\
\hline
\end{tabular}

Para el 50\% de los procesos se solicita con anterioridad al 95 día de la baja, tiempo bastante por encima de la duración estándar establecida por el INSS para la mayoría de los diagnósticos, más si tenemos en cuenta la duración para el 25\% de los procesos (percentil 25).

- Del total de los procesos, terminaron en Incapacidad Permanente 107 (1,8\%)

El 64,5\% de las altas por Incapacidad Permanente (69) se dieron a mujeres.

Tras realizar los contrastes de hipótesis (test ji-cuadrado de Pearson) para comparación de porcentajes entre variables, obtenemos dependencias entre las variables incapacidad permanente y medico de control ( $\mathrm{p}<0,05)$. El 93,5\% (100) de las incapacidades permanentes son solicitadas por médicos de control.

El 1,9\% (2) por psiquiatras y un 4,7\% (5) por psicólogos.

Tras realizar los contrastes de hipótesis (test ji-cuadrado de Pearson) para comparación de porcentajes entre variables, obtenemos dependencias entre las variables 
incapacidad permanente y recaída y, por otro lado, incapacidad permanente y tipo de pago $(\mathrm{p}<0,05)$. recaída.

De los procesos con Incapacidad Permanente, 31 (29\%) habían sido procesos con

El 85\% (91) de los procesos con alta por Incapacidad Permanente correspondían a procesos en pago directo.

El 86,9\% (93) de los procesos con alta por Incapacidad Permanente correspondía a procesos en pago por cuenta ajena.

Por lo que tendríamos 91 (85,04\%) procesos en pago directo, de los que 77 (84,61\%) corresponderían a pago por cuenta ajena.

El mayor número de altas por Incapacidad Permanente se producen en los diagnósticos más frecuentes y para los que se encuentra la mayor frecuencia en la relación con otras variables; Reacción de adaptación (28,03\%), R.A. con características emocionales mixtas (23,26\%) y R.A. con humor de ansiedad (13,08\%). No obstante, considero importante tener en cuenta que un $9,34 \%$ de todas las altas por Incapacidad Permanente son debidas a Reacción depresiva prolongada (309.1).

Tabla XII. Número de altas por Incapacidad Permanente en los diferentes Códigos diagnósticos

\begin{tabular}{llr}
\hline & \multicolumn{1}{c}{ Código diagnóstico } & Alta por I.P. \\
\hline 309 & Reacción de adaptación & 31 \\
309.4 & R.A. con alteración de emociones y conducta & 12 \\
309.8 & Otras reacciones de adaptación especificadas & 1 \\
309.81 & Trastorno por estrés postraumático prolongado & 2 \\
309.82 & R.A. con síntomas físicos & 1 \\
309.83 & R.A. con retraimiento & 1 \\
309.89 & Otra R.A. especificada NCOC & \\
309.9 & R.A. no especificada & 4 \\
309.0 & Reacción depresiva leve & 3 \\
309.1 & Reacción depresiva prolongada & 10 \\
309.2 & R.A. con perturbación de otra emoción & 1 \\
309.21 & Trastorno de ansiedad por separación & 14 \\
309.24 & R.A. con humor de ansiedad & 25 \\
309.28 & R.A. con características emocionales mixtas & \\
309.29 & Otra R.A. con perturbación de otras emociones & Total \\
309.3 & R.A. con alteración predominante de conducta & 107 \\
\hline & &
\end{tabular}

\section{DISCUSIÓN}

El mayor número de procesos diagnosticados en este periodo para esta patología corresponden a mujeres. En ellas es también en las que se produce un mayor número de recaídas y el mayor número de altas por Incapacidad Permanente. También estas dos situaciones se relacionan, de manera que, de los procesos que terminaron en Incapacidad Permanente, un tercio había sufrido recaída. Estos datos son importantes a la hora de realizar el control y seguimiento de estos procesos de cara a conseguir su resolución satisfactoria en el tiempo adecuado.

La edad en la que mayor número de trabajadores presentan esta patología son los 35 años. En las edades comprendidas entre 35 y 39 años es donde se presentan el mayor número de procesos, ampliándose en frecuencia hasta los 44 . Realmente, si tenemos en 
cuenta la edad de incorporación de las personas al trabajo en los últimos años, podemos decir que se trata de una patología con mayor incidencia en personas con una vida laboral corta, menos la Reacción de adaptación con humor de ansiedad que se presenta con mayor frecuencia en la década anterior, es decir en edades aún más jóvenes.

La coincidencia de los datos en sexo y edad se repite en varios de los estudios revisados $^{11}$.

Un dato a tener en cuenta es que en los mayores de 65 años la duración de la mayoría de sus procesos es superior al año.

Para el 44\% del total de los procesos, el no detallar ninguna especificación sintomática que lo clasifique en un subtipo diagnóstico determinado y no sólo como Reacción de adaptación, hace que se pierda mucha información acerca del diagnóstico específico en el global del diagnóstico y en la relación con el resto de las variables. Los subtipos diagnósticos (con el genérico Reacción de adaptación) son los que se repiten en frecuencia y también en casi todos los resultados al relacionarlos con otras variables: recaídas, edades más frecuentes de diagnóstico, número de propuestas de alta, procesos atendidos por médico de familia, psiquiatra o psicólogo. Sin embargo, no son los subtipos diagnósticos que mayor duración en situación de incapacidad presentan, sino el 309.82 R.A. con síntomas físicos, el 309.29 R.A. con perturbación de otras emociones; trastornos en los que puede incidir en mayor grado la indiferenciación diagnóstica o la comorbilidad, seguidos por el 309.81 Trastorno por estrés postraumático prolongado y 309.1 Reacción depresiva prolongada, para los que sí están descritos duraciones más altas en los tiempos estándar del INSS.

No parece que un determinado tipo de ocupación de los trabajadores esté más relacionada con esta patología en nuestra muestra de evaluación. Los grupos, además, no son equiparables en cuanto a sus características de cualificación laboral.

En Santa Cruz de Tenerife y Las Palmas de G.C. (enclaves de nuestros centros en Canarias) el diagnóstico de esta patología es muy frecuente. Una explicación podría ser el mayor número de desplazados por motivos laborales que residen allí y el alto número de inmigrantes. Llama la atención también, y en principio sin ninguna explicación que justifique estos resultados, la poca frecuencia diagnóstica de esta patología en Galicia, a pesar de que el porcentaje de patología psiquiátrica con relación al total e procesos controlados es mayor al $12 \%$ en todas las provincias. Un porcentaje muy importante de procesos es llevado por médicos de control $(81,9 \%)$, pero no disponemos de mucha información para sacar conclusiones respecto a los llevados por psiquiatras (sobre todo, tan solo 80 procesos) o por psicólogos. Además, los pacientes pueden ser derivados a especialistas ajenos o por nuestros médicos o por los del Servicio Público de Salud. Para poder llegar a alguna conclusión efectiva sería necesario analizar todos estos determinantes.

En un estudio realizado por médicos de otra Mutua ${ }^{11}$ relativo a la influencia del peritaje en la duración de la IT en la patología psiquiátrica más frecuente en contingencias comunes, en el que incluyen específicamente los trastornos de adaptación, los resultados les llevan a cuestionarse si está justificada la derivación de los pacientes a un especialista externo y concluyen que no lo está.

Coincide plenamente con los resultados de este estudio, ya que la duración media de procesos controlados por psiquiatras es mayor a la de los controlados por médico de control y psicólogos.

La explicación puede estar en la derivación a los psiquiatras de los pacientes con sintomatología más intensa y procesos más difíciles de controlar.

El número de recaídas de los procesos se relaciona directamente con la mayor frecuencia de la mayoría de las variables analizadas: sexo, edad, código diagnóstico, altas por Incapacidad Permanente y provincias con mayor número de procesos con este diagnóstico. 
El $77 \%$ de las altas por Incapacidad Permanente corresponden a procesos en pago directo cuenta ajena. Cuando un proceso supera el año de duración y pasa a ser evaluado por el INSS, pasa a esta situación.

El año de mayor número de bajas fue 2008, progresivamente han ido bajando en 2009 y 2010 por posible efecto de la crisis económica. Realmente hay menor número de trabajadores afiliados a la Mutua por el cierre de muchas empresas y el ajuste de personal laboral en las mismas, por lo que la posibilidad del desempleo ha llevado consigo la disminución del absentismo. En España, el absentismo laboral en 2007 era del 4,10\% y en el 2010 un 3,60\% según el Estudio de evolución del absentismo por países (UE) $2007 / 2010$ de Price Waterhouse Coopers, S.L.

No obstante, la previsión de reajuste de personal en las empresas con la posibilidad de pasar a la situación de desempleo también provoca el aumento de bajas laborales por esta patología ${ }^{12}$.

Aunque la duración media para el porcentaje mayor de los procesos $(18,2 \%)$ es de 31 a 61 días, el 52,6\% del total llega a hasta los 120 días. La duración de cada subtipo diagnóstico supera con mucho los Tiempos Estándar del INSS. En este aspecto existe un marco de mejora importante, en el que pueden estar implicadas alguna s de las variables del propio proceso: mejora diagnóstica y, por lo tanto, tratamiento adecuado, aumento de las propuestas de alta y solicitud más temprana en la evolución el proceso ${ }^{13}$.

Aumenta cada año el número de propuestas de alta solicitadas en relación con el número de procesos controlados. En muchos procesos hay que solicitarla de forma reiterada. Es posible que esté relacionado con la falta de criterios diagnósticos claros para encuadrar esta patología y su evolución, como hemos venido mencionando, no considerando la Inspección del S.P.S. el alta con nuestra propuesta.

La solicitud de propuesta de alta no se ajusta al tiempo estándar de duración de los procesos más diagnosticados según el INSS, por lo que no contribuyen todo lo que sería deseable a la disminución de la duración de los procesos.

Consideramos que es importante no demorar los procesos realizando las actuaciones necesarias precozmente con el objeto de evitar situaciones de renta.

Tanto el DSM-IV (APA 2001) como la CIE -10 (OMS 1992) determinan que el diagnóstico de trastorno adaptativo está enmarcado en la evaluación de ciertas variables: forma, contenido y gravedad de los síntomas, antecedentes y personalidad, y suceso o situación de la vida cotidiana. A pesar de los esfuerzos por especificar sus criterios, la práctica clínica pone de manifiesto que siempre ha sido objeto de polémica por conservar, hasta nuestros días, una definición diagnóstica vaga y residual ${ }^{14}$.

En esta línea, Casey y Dowrick opinan que dos aspectos ambiguos definen el diagnóstico de los trastornos adaptativos. Destacan la diferenciación imprecisa de sus manifestaciones y las reacciones esperadas ante la adaptación y la superposición del diagnóstico respecto a otros trastornos ${ }^{14,15}$.

En la misma línea, Batlle considera a los Trastornos de adaptación como una categoría intermedia entre la adaptación normal y un trastorno mental diagnosticable específico. Esta condición intermedia y la falta de toda lista específica de síntomas pueden facilitar la presencia de problemas en el momento de categorizar el diagnóstico ${ }^{8}$.

Seguramente esto influye a la hora de asignar la mayoría de los procesos una categoría diagnóstica concreta dependiendo del síntoma predominante y al intentar analizar algunos resultados con este síntoma como diferenciación, se tropieza con la numerosa proporción de procesos sin codificación detallada, sólo adscritos al diagnóstico genérico Reacción de adaptación.

Aun así, considero que la información que proporciona los resultados obtenidos puede ser útil en el futuro para mejorar el control y seguimiento de los trabajadores afectados por esta patología, ya que muchos de ellos reflejan un marco de mejora 
estimable. No obstante, los resultados de este estudio no pueden considerarse extrapolables a la totalidad de la población con esta patología, ya que la población estudiada corresponde a trabajadores de empresas afiliadas a Ibermutuamur, por lo que no son representativos de la población general.

Los factores socioeconómicos pueden ser más importantes a la hora de determinar la duración de la Incapacidad Temporal que incluso la propia patología, por lo que es conveniente realizar una entrevista clínica detallada que tenga en cuenta todos los aspectos (socio-familiares, laborales, económicos...) que pueden condicionar el inicio y el mantenimiento del paciente en esta situación.

Por otro lado, dentro de la categoría de los Trastornos de adaptación también se incluyen problemas cada vez más frecuentes como los relacionados con el acoso ("mobing"etc.), o el "síndrome del quemado" ("burn out"), etc., que se consideran "formas extremas de la sintomatología propia de los trastornos de adaptación" (GómezMacías et al., 2006) ${ }^{16}$.

Además, sería importante revisar los criterios diagnósticos de esta patología de forma que pueda delimitarse claramente, y conseguir una correcta adjudicación diagnóstica de los pacientes, con el consiguiente ajuste del tratamiento y el acortamiento de la incapacidad laboral que en estos momentos acarrean.

\section{CONCLUSIONES}

A tenor de los resultados y del análisis realizado, no hay ninguna variable que por sí misma pueda determinar la toma de decisiones para mejorar la gestión de la incapacidad laboral por esta patología, pero quizá sí la confluencia de varias de ellas pueda orientar a su gestión, ya que pueden tenerse en cuenta los datos que marcan la frecuencia en mujeres y en ellas, su mayor frecuencia de recaídas y evolución a incapacidad permanente, en edades comprendidas entre los 35 y 39 años.

Deberían incrementarse los programas específicos para ellas, de detección precoz, valoración, seguimiento y tratamiento de esta patología. Así podría minimizarse el impacto en su calidad de vida y en su vida laboral.

Es necesario codificar los procesos de acuerdo al síntoma predominante, ya que, no hacerlo, supone una pérdida importante de información para sacar conclusiones generales de impacto en la gestión, ya que considero que ha sido el inconveniente mayor a la hora de la explotación de datos de la muestra estudiada.

Realmente un mayor y mejor registro de los mismos permitiría entender mejor la relación existente entre los distintos factores o variables y la Incapacidad Temporal.

Así mismo, la adscripción correcta de los pacientes al diagnóstico de Trastorno de adaptación y su identificación respecto a la sintomatología predominante que presentan en el contexto del mismo, permitirá realizar el tratamiento más adecuado, lo que va a determinar la evolución de esta patología ajustándola al tiempo de recuperación funcional suficiente para el desempeño de los requerimientos o tareas profesionales del trabajador, evitando la permanencia excesiva en situación de incapacidad temporal que en ocasiones esconde problemas de otra índole.

\section{REFERENCIAS BIBLIOGRÁFICAS}

1. Vilariño Rodríguez DM, Vicaria Arroyo FJ. Trastornos de ansiedad-depresión en contingencia común en la ciudad de Málaga. Máster Universitario en MedicinaEvaluadora-Edición 2009. Instituto de Formación Continua. Universidad de Barcelona - Asepeyo; 2010.

2. Estrategia en Salud Mental del sistema Nacional de salud 2009-2013. Ministerio de Sanidad, Política Social e Igualdad. Sanidad 2011. 
3. Duración de la Incapacidad Laboral asociada a diferentes patologías en trabajadores españoles. Investigación financiada al amparo de lo previsto en la Orden TIN/190272009 de 10 de junio (premios FIPROS, Fondo para el Fomento de la Investigación de la Protección social).

4. Absentismo laboral en tiempos de crisis. PIMEC: Pequeña y Mediana Empresa de Cataluña. 2011.

5. Clasificación de enfermedades mentales de la American Psychriatric Association (APA) DSM IV-TR Disponible en http://www.psicoarea.com//dsm iv.htm

6. CIE 10. Disponible en http//www.psicomed.net/cie 10/cie10_indice.html

7. Gutiérrez Miras MG, Peñas Martínez L, Santiuste de Pablos M, García Ruipérez D, Ochotorena Ramírez MM, San Eustaquio Tudanca F, Cánovas Martínez M. Comparación de los sistemas de clasificación de los trastornos mentales CIE 10 y DSM IV. Atlas VPM 2008: 5:220-222.

8. Batlle Vila S. Trastornos de Adaptación. Universidad Autónoma de Barcelona 2009.

9. Tiempos Estándar de Incapacidad Temporal. Instituto Nacional de la Seguridad Social; 2009.

10. Official Disability Gudelines. Work-loss Data Institute. 12 ed.; 2007.

11. Almarcha Vicente MI, Aparicio Navarro JV, Enguix Monzó V. Influencia del peritaje en la duración de la IT en la patología psiquiátrica más frecuente en contingencias comunes. Máster Universitario en Medicina Evaluadora-Edición 2006-2007. Instituto de Formación Continua. Universidad de Barcelona - Asepeyo; 2010.

12. Rodríguez Ortiz de Salazar B, Gómez Gálligo A, García Parra A, López Gómez I. Sintomatología depresiva asociada al trabajo como causa de incapacidad laboral en la Comunidad de Madrid. Mapfre medicina 2005; 16:184-194.

13. Baumeister $\mathrm{H}$, Kufner $\mathrm{K}$. It is time to adjust the adjustment disorder category. Current Opinion in Psychiatry 2009; 22:409-412.

14. Herrero Gómez V, Cano Vindel A. Un caso de trastorno adaptativo con ansiedad: evaluación, tratamiento y seguimiento. Anuario de Psicología Clínica y de la Salud 2010; 6:53-59.

15. Giovanni Carta M, Balestrieri M, Murru A, Hardoy MC. Adjustment Disorder: epidemiology, diagnosis and treatment. Clínica Practic and Epidemiology in Mental Health; 2009.

16. Baños Rivera RM, Guillén Botella V, Botella Arbona C, García Palacios A, Jorquera Rodero M, Quero Castellano S. Un programa de tratamiento para los trastornos adaptativos. Un estudio de caso. Apuntes de Psicología 2008; 26:303-316.

17. Guía de valoración de incapacidad laboral para médicos de atención primaria. Escuela Nacional de Medicina del trabajo. Instituto de Salud Carlos II. Ministerio de Ciencia e Innovación. 2010.

18. Casey P. Adjustment Disorder: Epidemiology, Diagnosis and Treatment. CNS Drugs 2009; 23:927-938.

19. Incapacidad temporal por patología psiquiátrica en un centro de salud. Centro de Salud Urbano Dr. Pujol i Capsada. El Prat de Llobregat -Barcelona). 2000.

20. Dobricki M, Komproe IH, de Jong JTVM. Adjustment disorders after severe life-events in four postconflict settings. Social Psychiatry and Psychiatric Epidemiology 2010; 45:39-46.

21. Herrero Gómez V, Cano Vindel A. Un caso de trastorno adaptativo con ansiedad: evaluación, tratamiento y seguimiento. Anuario de Psicología Clínica y de la Salud 2010; 6:53-59.

22. Vázquez Díaz AJ, Pérez Cano D, Moreno Ramos R, Arroyo Yanes F, Suárez Delgado JM. Factores psicosociales motivacionales y estado de salud. Med Segur Trab 2010; 56:12-21.

23. Baumeister H, Harter M. Prevalence on mental disorders based on general population surveys. Soc Psychiatry Psychiatr Epidemiol 2007; 42: 537-46.

24. Laugharne J, van der Watt G, Janca A. It is too early for adjusting the adjustment disorder category. Curr Opin Psychiatry 2009; 22:50-54 


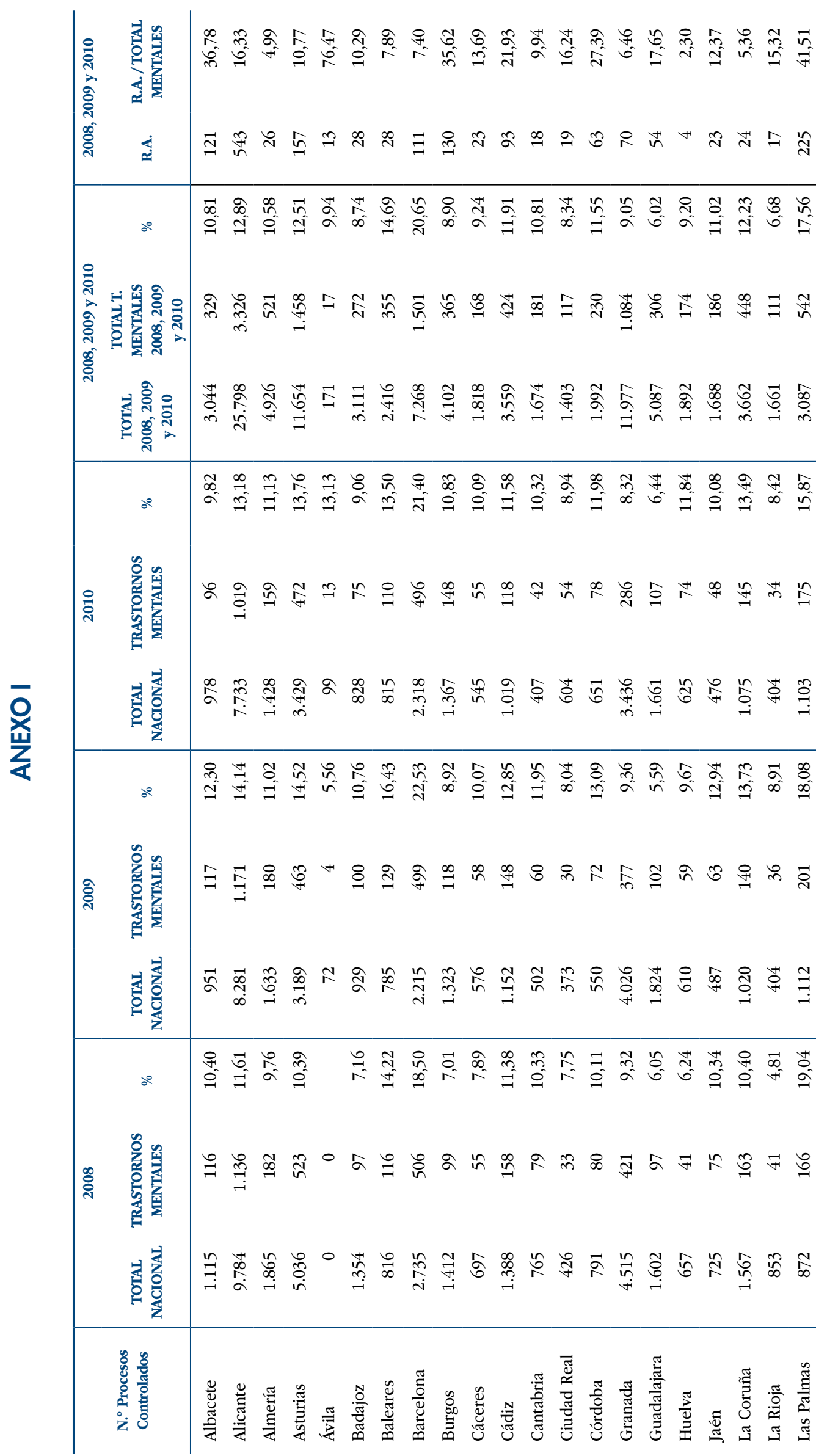




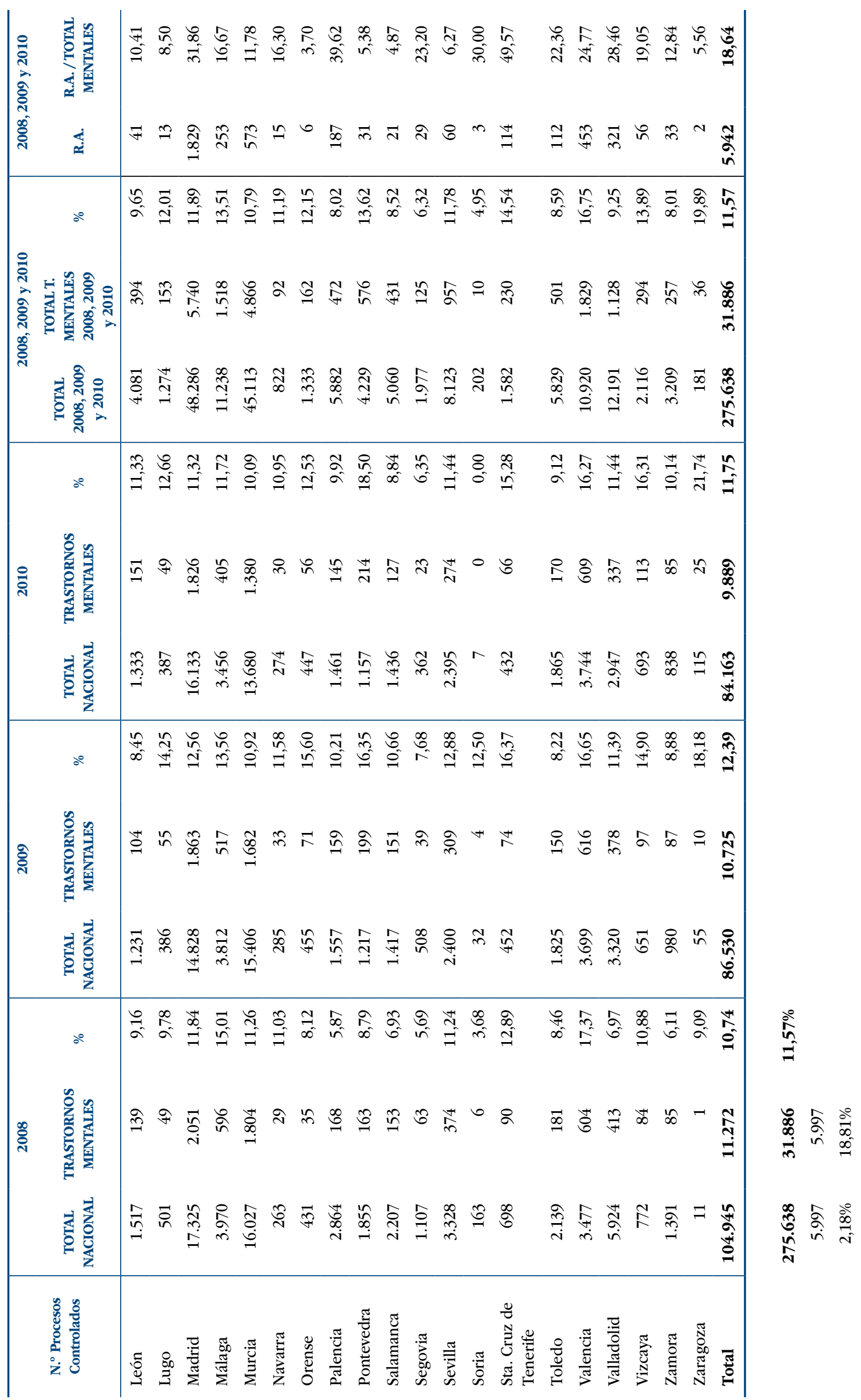

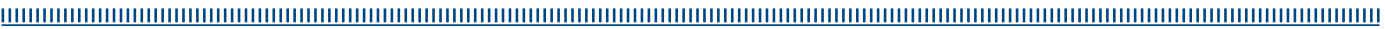

